\title{
En busca de protección a las puertas de Europa: refugiados, etiquetado y prácticas disuasorias en la frontera sur española
}

\section{Seeking protection at the gates of Europe: refugees, labeling and dissuasion practices at the southern Spanish borders}

Recibido el 13 de enero de 2020 Aceptado el 4 de mayo de 2020. Publicado el 8 de mayo de 2020.

*Autora para correspondencia: Ana López-Sala, correo electrónico, ana.Isala@cchs.csic.es
Esta obra está protegida bajo una Licencia Creative Commons Atribución-NoComercial 4.0 Internacional.
Ana López-Sala ${ }^{a \star}$ (D) https://orcid.org/0000-0003-2756-7042 Gracia Moreno-Amador ${ }^{a}$ (i) https://orcid.org/0000-0002-7711-3914

a Consejo Superior de Investigaciones Científicas (CSIC), Instituto de Economía, Geografía y Demografía, Madrid, España, correo electrónico: ana.lsala@cchs.csic.es, gracia.moreno@cchs.csic.es

\section{Resumen:}

A partir de un análisis que subraya las prácticas de contención del acceso de los refugiados desde el denominado paradigma disuasorio, este artículo usa la literatura del etiquetaje para investigar la gestión del refugio en la frontera sur española a lo largo de las últimas tres décadas. Además de detenernos en algunos elementos del marco regulatorio del refugio en España y su evolución, el análisis presentado se basa en 34 entrevistas en profundidad semiestructuradas realizadas entre 2016 y 2019 en Madrid, Cádiz, Ceuta y Melilla a diferentes actores públicos y miembros de organizaciones sociales, nacionales e internacionales, implicados en la gestión del refugio en España. Las conclusiones del análisis indican que el etiquetado de la inmigración africana como una inmigración exclusivamente laboral ha moldeado la gestión política del refugio en la frontera sur española a través de prácticas que han impedido, limitado y disuadido el acceso de potenciales solicitantes de asilo al sistema español de protección internacional. Por ello la gestión del refugio ha quedado ampliamente supeditada al control migratorio.

Palabras clave: refugiados, etiquetado, paradigma disuasorio, frontera, control migratorio, España.

\section{Abstract:}

Based on an analysis on the practices that have become generalized over the past few decades to limit refugees from entering the territory through what has been dominated the dissuasion paradigm, this article uses the literature on labeling to research how asylum has been managed at Spain's southern border over the

CÓMO CITAR: López-Sala, A. \& Moreno-Amador, G. (2020). En busca de protección a las puertas de Europa: refugiados, etiquetado y prácticas disuasorias en la frontera sur española. [Seeking protection at the gates of Europe: refugees, labeling and dissuasion practices at the southern Spanish borders]. Estudios Fronterizos, 21, e048. https://doi.org/10.21670/ref.2006048 
past three decades. The analysis is based in 34 in-depth interviews conducted between 2016 and 2019 in Madrid, Cádiz, Ceuta and Melilla to members of different public institutions and social organizations involved in the implementation of refugee policies at the Southern Spanish border. The "labeling" of African immigrants as labor migrants (or as "bogus" asylum seekers) has shaped the refugee policies at this border resulting in practices of contention and dissuasion of the flows and restrictions in the access to the Spanish protection system under the priority of the logic of control.

Keywords: refugees, labeling, dissuasion paradigm, border, migration control, Spain.

\section{Introducción}

A pesar de su condición de frontera exterior de la Unión Europea y de su atractivo como país de destino de flujos migratorios, España ha sido uno de los países europeos que ha recibido un menor número de solicitudes de protección internacional a lo largo de las últimas décadas. Según los datos proporcionados por eurostat, entre 1998 y 2014 las solicitudes realizadas en España no superaron 1.5\% del total de la Unión Europea, un porcentaje muy inferior al de países con mayor tradición receptora - como Alemania, Francia, Suecia y Gran Bretaña- y al de otros países europeos localizados en la frontera sur - como Grecia o Italia. Al limitado número de solicitudes se une otro rasgo singular del régimen español de asilo y es lo que podemos denominar su "desviación territorial". Así, a pesar de su localización geográfica en los confines del espacio europeo y de su proximidad con el continente africano las solicitudes de protección internacional se han realizado fundamentalmente en el interior del territorio y no en las fronteras marítimas y terrestres periféricas. Entre 1995 y 2014, según los datos proporcionados por el Ministerio del Interior, solo $14.26 \%$ del total de solicitudes de refugio se presentaron en las ciudades fronterizas de Ceuta y Melilla; ${ }^{1}$ mientras que en 2006, durante la denominada "crisis de los cayucos", solo se realizaron en Canarias 366 solicitudes (Alto Comisionado de las Naciones Unidas para los Refugiados [ACNUR], 2009), a pesar de que en ese año llegaron a las costas de esta región del archipiélago más de 32000 personas a bordo de precarias embarcaciones. ${ }^{2}$

\footnotetext{
${ }^{1}$ Desde 2005 solo se dispone de datos agregados sobre la distribución de las solicitudes por lugar de solicitud (distinguiendo entre solicitudes realizadas en frontera y en el interior del territorio), sin especificar la provincia fronteriza en la que se ha realizado. Solo se disponen de datos específicos para el periodo 1995-2018 en el caso de las ciudades de Ceuta y Melilla.

${ }^{2}$ Adicionalmente es importante destacar que todas estas solicitudes se realizaron en el interior del territorio del archipiélago, no en frontera marítima, generalmente, como en su momento indicaron diversos informes y algunas de las personas entrevistadas durante esta investigación que se encontraban en los centros de internamiento de inmigrantes (Comisión Española de Ayuda al Refugiado [CEAR], 2007; ACNUR, 2009).
} 
Este escaso número de solicitudes en comparación con otros países europeos ${ }^{3}$ ha sido objeto, durante las últimas tres décadas, de una amplia discusión entre los especialistas que han destacado entre otras razones el escaso atractivo de España como país donde buscar protección por la deficiencia de los instrumentos e iniciativas dirigidas a la acogida, el elevado porcentaje de resoluciones negativas o la aplicación, durante un amplio periodo, del denominado procedimiento de inadmisión a trámite (Gil-Bazo, 1998; Jubany, 2002; Fullerton, 2005; Santaolaya, 2006). Otros autores han esgrimido, además, que las dificultades para obtener la condición de refugiado y la existencia de vías alternativas de acceso al territorio y al mercado de trabajo en el caso español tuvieron el efecto de "desviar" a muchos potenciales solicitantes de asilo hacia el régimen de extranjería (Izquierdo, 2001, 2004). ${ }^{4}$ La escasez de las cifras ha tenido un efecto en el imaginario español que permea las visiones institucionales y de los actores sociales que participan en la gestión de los flujos y la implantación de las políticas de acogida. Como afirmó recientemente Garcés-Mascareñas, España llegó a ser un país de inmigración que nunca se reconoció como un país de asilo (GarcésMascareñas, 2019).

Su peculiar distribución territorial, sin embargo, apenas ha despertado atención entre los especialistas. Pero ¿cuáles son las razones que explican este bajo número de solicitudes de protección internacional en las fronteras españolas, especialmente aquellas más próximas al continente africano?; ¿cuáles han sido las especificidades de la gestión del refugio en frontera en el caso español?; ¿cómo ha sido gestionado el refugio en el contexto de la conformación de la política española de control migratorio y del régimen fronterizo europeo?

La intensidad de los flujos migratorios hacia España a lo largo de las últimas décadas y el carácter prioritario que ha llegado a adquirir la "contención migratoria" en la política ha centralizado los análisis en la gestión fronteriza de la inmigración irregular (FerrerGallardo, 2008; Godenau, 2014; Ferrer-Gallardo \& Gabrielli, 2018; Godenau \& LópezSala, 2016; López-Sala \& Godenau, 2017a). Por el contrario, el escaso peso demográfico de las solicitudes de protección internacional ha alejado la cuestión del refugio del interés académico, también en el caso de los estudios fronterizos. Más recientemente la

\footnotetext{
${ }^{3}$ Es necesario destacar, sin embargo, que este patrón ha cambiado enormemente en el último lustro, no así la señalada pauta de "desviación territorial". Desde la denominada "crisis de los refugiados", el número de solicitudes de asilo en España se ha incrementado de forma muy significativa año tras año hasta llegar a un máximo histórico en 2019. Los números pueden ser más expresivos: mientras que en 2014 el número de solicitudes fue de 5 952, en 2019 ascendió a 118 264, según los datos proporcionados por el Ministerio del Interior. Esto ha convertido a España, por primera vez en su historia reciente, en uno de los principales países de destino de refugiados en Europa. En 2019, según datos de Eurostast, España ocupaba la tercera posición solo por detrás de Alemania y Francia. En la actualidad la mayor parte de los solicitantes proceden de Venezuela, Colombia y países centroamericanos (Honduras, Nicaragua y El Salvador). A pesar de todas estas transformaciones se mantiene el bajo número de solicitudes realizadas en frontera (en 2019 solo $6 \%$ del total) y la escasa presencia de ciudadanos africanos entre los solicitantes (véase CEAR, 2019; véase también https://www.cear.es/solicitar-asilo-en-fronteras/).

${ }^{4} \mathrm{~A}$ principios de la década de 2000 Antonio Izquierdo indicó que la primacía de un modelo de regulación migratoria laboral, construido y diseñado para responder a las necesidades del mercado de trabajo, permitió vías de acceso y de estabilización legal a través del régimen general de extranjería a los potenciales solicitantes de asilo. Su análisis sobre los procesos de regularización mostraron lo que denominó el "efecto dominó" de las políticas migratorias entre las distintas categorías de flujos; en concreto cómo muchos potenciales solicitantes de asilo entraron en el territorio con visados de turista de corta duración, posteriormente residieron en situación irregular y accedieron posteriormente a un permiso de trabajo o residencia a través de las regularizaciones extraordinarias o del procedimiento de arraigo (Izquierdo, 2001, 2004).
} 
denominada "crisis de los refugiados", la llegada a España de ciudadanos sirios huyendo de la guerra y los acuerdos adoptados en el contexto europeo sobre reasentamiento han impulsado los análisis sobre las políticas españolas de acogida y sus deficiencias (Iglesias y Estrada, 2018; Iglesias et al., 2018; Garcés-Mascareñas, 2019; Garcés-Mascareñas \& Moreno-Amador, 2019). En definitiva, hasta la fecha, la gestión del refugio en las fronteras españolas ha despertado escasa atención entre los especialistas, a pesar del protagonismo que ha adoptado recientemente en el caso de otras zonas del perímetro europeo (McMahon \& Sigona, 2018; Vradis et al., 2019).

A partir de un análisis que subraya las interconexiones y vínculos dinámicos entre los regímenes de gestión de la movilidad en espacios fronterizos y la literatura reciente sobre la generalización durante las últimas décadas de las prácticas de contención del acceso de los refugiados a través de lo que se ha denominado el "paradigma disuasorio" (Gammeltoft-Hansem, 2011a; Gammeltoft-Hansen, 2011b; Gammeltoft-Hansen \& Tan, 2017; Agier, 2013; López-Sala, 2015a; 2020; Fitzgerald, 2019; Collyer, 2019; Giuffré \& Moreno-Lax, 2017) este artículo usa la literatura del etiquetaje (conocida en inglés como la teoría del labeling) para investigar las prácticas de gestión del refugio en las fronteras terrestres periféricas y marítimas españolas en las últimas décadas. Estas dos aproximaciones, y su imbricación, enmarcan teóricamente el desarrollo del artículo. Nuestra principal tesis es que bajo el "etiquetado" de la inmigración que trascurre por la frontera sur española, fundamentalmente africana, como un flujo de carácter exclusivamente económico y, por tanto, como una movilidad voluntaria, la articulación e implantación de la política española en el contexto de la conformación del régimen fronterizo europeo ha impedido, limitado y disuadido el acceso de potenciales solicitantes de asilo al sistema español de protección internacional. La construcción social y política del migrante africano como paradigma de la movilidad motivada por causas económicas, y, por tanto, su etiquetado como "inmigrante ilegal" o "falso solicitante de asilo" ha impregnado la intervención en las fronteras españolas con una lógica que prima el principio de contención y descarta las necesidades y los derechos de protección (Leach \& Zamora, 2006). Por ello la gestión del refugio ha quedado ampliamente supeditada al control migratorio al adoptar rasgos que confirman, pero también amplían, las categorías presentadas por otros autores dentro del denominado "paradigma disuasorio" (Gammeltoft-Hansen \& Tan, 2017). Mientras que la mayor parte de los autores que han descrito este nuevo paradigma de gestión del refugio consideran que los Estados han puesto en práctica estas políticas para circunvalar sus obligaciones legales con la jurisdicción nacional e internacional (Gammeltoft-Hansen \& VedstedHansen, 2016), la aplicación de estas medidas en el caso español responde además a la construcción de este tipo de migración como una migración económica a la que el Estado no debe protección. De ahí el encaje del régimen de refugio y de extranjería en una misma lógica, la de la lucha contra la inmigración irregular y la necesidad de realizar un análisis particular de la gestión del refugio "desde la frontera".

Los efectos de este etiquetado en la categorización, clasificación y jerarquización de los flujos que transitan a través de estos espacios liminares ahondan en su papel como mecanismos de filtrado selectivo (Godenau \& López-Sala, 2016) en este caso a través de la construcción del contorno de aquellos considerados receptores legítimos de protección. La plasmación política de esta estructuración jerárquica y clasificatoria de los migrantes, según lo que podemos denominar "su nivel de merecibilidad", se ha manifestado de forma particularmente expresiva con las llegadas a través de la frontera sur de nacionales procedentes de Siria etiquetados, por las autoridades europeas y 
españolas —los actores de la gestión y la opinión pública—, como refugiados legítimos (Valles, 2016; Amnistía Internacional, 2016).

El análisis presentado en este artículo se basa en 34 entrevistas en profundidad semiestructuras realizadas a diferentes actores públicos y miembros de organizaciones sociales, nacionales e internacionales, implicados en la gestión del refugio en España. Entre los entrevistados se incluye a técnicos de la Oficina de Asilo y Refugio (la agencia encargada de esta materia dependiente del Ministerio del Interior), responsables de las principales organizaciones sociales implicadas en la atención de solicitantes de asilo en España, abogados y asesores jurídicos de diversas organizaciones, miembros de entidades sociales que han implantado programas de atención a los refugiados, de los cuerpos y seguridad del Estado que intervienen en la realización de las entrevistas a los solicitantes, responsables de organizaciones sociales que trabajan en la defensa de los derechos de los inmigrantes y miembros de colegios de abogados y expertos del ámbito jurídico. Las entrevistas se realizaron en Madrid (10), el Estrecho (la provincia de Cádiz) y en las ciudades de Melilla y Ceuta (24) entre 2016 y 2019 utilizando un guion que incluye preguntas abiertas y cerradas y en donde se ha intentado recoger de forma retrospectiva información sobre la gestión del refugio en distintos enclaves de la frontera sur española a lo largo de las últimas décadas.

El artículo se divide en dos partes principales. La primera parte se detiene en la literatura sobre el etiquetado, en sus formulaciones primigenias y su reelaboración más reciente, con el objetivo de enmarcar teóricamente el análisis y analizar la influencia de esta construcción en el diseño y aplicación de esta política en el caso español a través de la información extraída del trabajo de campo. La segunda parte, encajada en los estudios que han analizado las políticas de refugio desde la perspectiva del denominado paradigma disuasorio, profundiza en las prácticas políticas y administrativas que han impedido el acceso al territorio, el acceso al procedimiento y, más recientemente, la limitación de la movilidad intraterritorial de los solicitantes de asilo que transitan a través de los corredores de la frontera sur española. Para ello no solo se emplean los resultados del trabajo de campo, sino que también se presentan de forma muy sucinta, como veremos, los cambios en la regulación (admisión a trámite y procedimientos específicos normativos en frontera) y en la intervención política (externalización, limitación de la movilidad intraterritorial de los solicitantes, etcétera). Este análisis pretende, por tanto, empezar a llenar una laguna en los estudios fronterizos y migratorios españoles a través del análisis de la gestión del refugio en frontera que nos permite, adicionalmente, presentar algunas claves para entender la desviación territorial y la escasez de solicitudes en las fronteras periféricas terrestres y marítimas españolas.

\section{Construyendo el contorno de los merecedores de protección}

\section{La teoría del etiquetado en los análisis sobre las políticas de refugio}

Aunque la teoría del etiquetado tiene una larga tradición, en las ciencias sociales ha experimentado un importante impulso como marco analítico en los estudios migratorios a lo largo de las tres últimas décadas, por lo que llega a ser considerada por algunos especialistas como una de las más significativas contribuciones de la sociología 
a los análisis sobre las migraciones forzosas (Stepputat \& Nyberg-Sorensen, 2014). Esta consideración es en parte resultado de la estela que en este campo han dejado algunos estudios realizados desde finales de la década de 1970. Wood definió el etiquetado como "una forma de referirse al proceso a través del cual las agendas políticas son establecidas y de forma más particular a cómo las personas, concebidas como objeto de la política, son definidas" (Wood, 1985, p. 348). Destacó también que el etiquetado es un atributo particularmente significativo de las burocracias y un medio importante del desempeño estatal. En sus propias palabras, "las etiquetas tienden a objetivar a las personas, desvinculándolas de su historia, transformándolas en casos estandarizados y vinculándolas con las instituciones que las administran" (Wood, 1985, p. 355; véase también Stepputat \& Nyberg-Sorensen, 2014). Ya en la década de 1980, Zetter examinó cómo y con qué consecuencias las personas eran etiquetadas como refugiados y cómo la identidad es formada, transformada y manipulada en el contexto de la formulación e implementación de estas políticas públicas (Zetter, 1991). En su análisis sostuvo que el etiquetado es un proceso de "estereotipación" que implica desagregación y diferenciación, estandarización, designación y la formulación de nítidas categorías. Más recientemente y en un contexto muy diferente al observado en los años de 1980, Zetter reformuló parte de su análisis y sostuvo que las nuevas dinámicas de la migración forzosa han producido una transformación del régimen del refugio y de la etiqueta "refugiado". En concreto ha sostenido que en la construcción del etiquetado prima en la actualidad la determinación de cómo se distribuye el estatuto de refugiado y cómo las prácticas institucionales buscan distinguir este estatuto de otras categorías de migrantes. En su opinión, la formación de la etiqueta de refugiado refleja la complejidad actual de las causas y los patrones de la movilidad humana. La transformación de este etiquetado, que responde a esta creciente complejidad, se ha visto representada por un proceso de "fraccionamiento" burocrático con el objetivo de gestionar estas nuevas y complejas dinámicas y en donde los Estados — del Norte- se han consolidado como los principales actores de la reformulación y modulación de estas categorías con base en sus propios intereses (Zetter, 2007).

Durante la última década y especialmente con la conocida "crisis de los refugiados" la investigación sobre el etiquetado ha retomado su centralidad en la investigación sociológica en el marco, por añadidura, de un intenso debate sobre las definiciones y la distinción entre migración forzosa y migración voluntaria. Diversos estudios han profundizado en cómo estas políticas del etiquetado han minado la estructura de protección en las políticas de refugio legitimando políticas restriccionistas y exclusionistas en los Estados receptores. Estos estudios han subrayado, por ejemplo, cómo estas construcciones establecen una clasificación de los individuos en movimiento basada en percepciones sobre quién es merecedor, o no, de estatutos de protección y revelando una dinámica compleja que impregna las políticas públicas a través de una distinción entre sujetos legítimos e ilegítimos. Así, Sajjad ha sostenido, por ejemplo, basándose en su estudio sobre los refugiados afganos, que "estas etiquetas, presentadas como categorizaciones neutras, ocultan el hecho de que son producto de procesos burocráticos". Además, sostiene, que "los procesos de etiquetado implican necesariamente relaciones de poder y pueden considerarse actos de gobernanza por parte de los Estados que con base en determinados intereses moldean y formulan diversas categorías en diferentes contextos y en diferentes momentos" (Sajjad, 2018, pp. 42 y 46). Otros autores han destacado cómo este etiquetado moldea un sistema de clasificación y ordenación de las personas en movimiento según categorías que tiene 
una destacada influencia sobre sus oportunidades de vida (Janmyr \& Mourad, 2018); cómo este etiquetado opera con base en los intereses del Estado y a expensas de los derechos de los potenciales solicitantes de asilo que transforma las políticas de refugio en una esfera política donde priman los mecanismos disuasorios y el incremento del control migratorio (McFadyen, 2016); o cómo la forma como se etiqueta, categoriza y diferencia a los migrantes ("aquellos que están en movimiento") tiene enormes implicaciones en el tipo de obligaciones morales y legales que los Estados y las sociedades de acogida consideran que tienen hacia ellos (Sigona, 2017).

La reciente crisis de los refugiados en Europa ha impulsado además un intenso debate académico sobre este etiquetado y la construcción de categorías. En concreto este debate se ha centrado en lo que Apostolova y posteriormente Crawley y Skleparis han denominado el "fetichismo de las categorías" con el argumento de que las categorías binarias dominantes - migrantes vs. refugiados - fracasan en capturar adecuadamente la complejidad de los determinantes políticos, sociales y económicos de la migración y su cambiante significado para los individuos a lo largo del tiempo y el espacio (Apostolova, 2015; Crawley \& Skleparis, 2018; Bivand Erdal \& Oeppen, 2018). Como Becker ha sugerido, los actores más poderosos, fundamentalmente los Estados, establecen y usan estas categorías - y sus etiquetas- para enmarcar un problema que refleja cómo ciertos temas son representados en los debates políticos y en el discurso público (Becker, 2014). Pero como indicó Moncrieffe, las categorías no solo representan o reflejan el mundo, sino que simultáneamente lo crean y lo limitan (Moncrieffe, 2007). Estas categorías, en definitiva, son resultado de las políticas y de las relaciones de poder que apuntalan lo que se ha denominado el proceso de delimitación —el proceso a través del cual estas categorías son construidas, el propósito al que sirven y sus consecuencias (Crawley \& Skleparis, 2018).

\title{
"No..., ellos no son refugiados...". De la protección de los refugiados a la protección de las fronteras
}

\begin{abstract}
Las personas que atraviesan los perímetros en Ceuta y Melilla son inmigrantes por razones económicas que, evidentemente, es una situación dramática (...), pero no les legitima para pedir asilo ni les legitima para entrar ilegalmente en nuestro país (declaraciones del ministro del Interior, Jorge Fernández Díaz; Europa Press, 10 de febrero de 2015).
\end{abstract}

El etiquetado de la inmigración africana que trascurre a través de los corredores marítimos -y terrestres periféricos- hacia España como una movilidad exclusivamente económica empezó a construirse entre finales de la década de 1980 y principio de la de 1990 de forma casi paralela a la transformación de España en país de inmigración y de su adhesión a los tratados europeos de Schengen y de Dublín. Esta simultaneidad explica en gran parte cómo este etiquetado ha moldeado la política migratoria y de refugio española en la frontera sur convertida en un instrumento al servicio de la contención de la movilidad, y en donde las medidas de protección han brillado por su ausencia. Existe un gran consenso entre nuestros entrevistados en torno a la idea de que la construcción de la inmigración africana como una movilidad exclusivamente económica supuso su identificación y categorización como un flujo 
ilegal e ilegítimo, que pretendía acceder a territorio español sin cumplir los requisitos que se establecían por el Estado. También fue retratada como una inmigración oportunista que empleaba la "vía del refugio" como un mecanismo facilitador de su acceso territorial y estatutario, que pretendía en última estancia aprovecharse del sistema, ${ }^{5}$ y cuya demanda, considerada fraudulenta, ponía en peligro el régimen de asilo y la protección de los "legítimos refugiados". Los efectos de esta construcción se transformaron en elementos articuladores de la política a partir, como indica la teoría del etiquetado, de una categorización, estereotipación y estandarización de todos los inmigrantes africanos como refugiados fraudulentos. ${ }^{6}$ La construcción de este tipo de movilidad como resultado de una decisión elegida, no forzosa, explica en gran parte la dinámica observada en las fronteras españolas desde principio de la década de 1990. Es también a principios de los años de 1990 cuando esta lógica de la contención de una inmigración considerada no merecedora de protección empezó a moldear la regulación del asilo en contraste con lo observado en la década anterior.

Es necesario subrayar que la primera ley española de asilo aprobada en 1984, en un momento en que España no se había convertido todavía en destino de flujos migratorios, estuvo en sintonía con el momento histórico que vivía a principios de la década de 1980. Tras décadas de dictadura, España firmó la Convención de Ginebra en los primeros años de su inaugurada democracia, en el mismo año que el derecho de asilo fue garantizado en la Constitución de 1978. Esta primera ley de asilo, regida por tres grandes principios orientadores - "la solidaridad, hospitalidad y tolerancia"contenidos en su exposición de motivos (Espada Ramos \& Moya Escudero, 1985), ha sido calificada en numerosas ocasiones como una ley extremadamente generosa en el contexto de las políticas restrictivas en esta materia desarrolladas en los países europeos desde los años de 1970 (Escobar-Hernández, 1992). Esta ley reconocía el derecho de entrada y residencia a los solicitantes durante todo el procedimiento y los protegía de la expulsión. Como sostuvo Gil-Bazo, los contenidos y orientación de esta ley fueron resultado de un contexto en el que primó el deseo del Estado español de implantar un sistema de asilo respetuoso con los derechos humanos y de responder ante la deuda histórica que supuso la acogida por otros países de más de tres millones de refugiados españoles durante el periodo franquista (Gil-Bazo, 1998). La reforma de la primera ley de asilo en 1994 — una fecha que coincide con el inicio de la construcción de las vallas en Ceuta y Melilla y del régimen de vigilancia en frontera marítima, y apenas dos años después de la imposición de visado para los nacionales de Marruecos- quiebra esta vocación y desarrolla una norma mucho más en sintonía con la nueva situación de España como perímetro exterior de Europa. En el debate público y político en torno a la reforma de la ley apareció, en los primeros años de la década de 1990, la idea de

\footnotetext{
${ }^{5}$ Esta percepción de los inmigrantes africanos como solicitantes de asilo fraudulento que pretendían aprovecharse del sistema fue también señalada por algunas investigaciones realizadas a principios de la década de 2000 (véase por ejemplo Jubany, 2002 y Leach \& Zamora, 2006).

${ }^{6}$ Los estudios sobre la política migratoria española también han señalado su carácter selectivo y en cierto sentido impregnado de tintes raciales a través de la delimitación de ciertas categorías étnicas y nacionales como categorías preferentes; en concreto la preferencia por la inmigración latinoamericana frente a la inmigración africana (Izquierdo et al., 2003). Aunque se trata de un tema controvertido algunos investigadores han subrayado este componente racial en la conformación de la política española (véase por ejemplo Vives, 2011). En nuestro trabajo de campo muchos de los entrevistados, aunque no emplean el término racismo, sí que han sostenido que ha existido discriminación hacia los migrantes africanos que solicitaban asilo, algo que han sostenido ha sido particularmente evidente durante la llegada de refugiados sirios considerados por la opinión pública y los responsables políticos como refugiados legítimos.
} 
que la ley anterior había sido empleada fraudulentamente como un mecanismo de acceso al territorio por inmigrantes económicos africanos que habían aprovechado las oportunidades que ofrecía esta puerta trasera (Pérez-Sola, 2011). En su exposición de motivos, la ley de asilo de 1994 indicaba la necesidad de adoptar las medidas necesarias para "impedir la utilización fraudulenta con fines de inmigración económica del sistema de protección a los refugiados". Este argumento fue incluso empleado en el preámbulo de la propia ley donde también se indicaba que:

el sistema de protección de los refugiados se veía desvirtuado en la práctica por un número creciente de solicitudes, en su mayoría de inmigrantes económicos (...), lo que dificultaba una acogida adecuada y provoca el consiguiente retraso en la resolución de las peticiones, convirtiéndose en la práctica en la principal vía de inmigración irregular hacia nuestro país (Ley 9/1994, 1994).

Esta visión se reflejó además en numerosos documentos legales y administrativos. La idea del uso abusivo del sistema de asilo se encontraba ya en la "Proposición no de Ley sobre la situación de los Extranjeros en España" de 1991 (Diario de Sesiones, Congreso de los Diputados, 9 de abril de 1991). La creación de la Oficina de Asilo y Refugio (OAR) en 1992 surge adicionalmente con un doble objetivo, tal y como señaló su entonces directora:

por un lado, mejorar la protección y el procedimiento de los expedientes de asilo que cumplían los requisitos para obtener el estatuto de refugiado; y, por otro lado, evitar la utilización fraudulenta de la vía de asilo y refugio por parte de inmigrantes económicos para evitar la aplicación de la normativa general en materia de extranjería (Bodelón, 1993, p. 47).

El análisis de las memorias de la OAR a lo largo de la década de 190 muestra precisamente la extensión de un clima de desconfianza ante los solicitantes de asilo, en especial los africanos, de las autoridades y los funcionarios, como también han puesto de manifiesto algunos estudios (Jubany, 2011).

A partir de este momento, la instalación de la lógica del bloqueo de la vía del refugio como un instrumento al servicio del control migratorio en la frontera sur ha imbuido el desarrollo posterior, tanto normativo, como administrativo, de forma similar a las dinámicas subrayadas por los teóricos del denominado paradigma disuasorio.

\section{En la interfaz de los regímenes de la movilidad fronteriza. Construyendo la gestión del refugio en tiempos de control migratorio}

A lo largo de los años la conformación del régimen fronterizo español ha incorporado un amplio abanico de instrumentos políticos que han incluido la aplicación de tecnología de barrera y de vigilancia a distancia, el control remoto, la cooperación bilateral y la externalización (López-Sala, 2015a; López-Sala \& Godenau, 2016, 2017b). Este régimen ha estado anclado en lógicas que concuerdan con el denominado "paradigma disuasorio". La literatura sobre el paradigma disuasorio se ha centrado en analizar las prácticas desarrolladas por los Estados, bien en solitario o en colaboración con otros, para restringir el acceso de los refugiados al sistema de asilo 
a través de múltiples mecanismos que incluyen, entre otros, el bloqueo del tránsito y el procesamiento deslocalizado, la prevención del acceso al territorio, del acceso al sistema de protección, o la disuasión selectiva del destino (Vedsted-Hansen, 1999; Hathaway \& Gammeltoft-Hansen, 2015; Hirsch, 2017; Gammeltoft-Hansen, 2011b; Gammeltoft-Hansen \& Tan, 2017; Giuffré \& Moreno-Lax, 2017; Fitzgerald, 2019). Los dos primeros mecanismos pueden ser considerados parte de las denominadas "prácticas de no entrada"7 (Hathaway, 1992) en referencia a los instrumentos políticos de externalización que dificultan la llegada al territorio de los Estados que pueden otorgar protección. ${ }^{8}$ Este aspecto fue ampliamente analizado por GammeltoftHansen en 2011 indicando que esta territorialización y externalización del refugio ha producido en última instancia una disputa o un litigio de protección (a "protection lite") (Gammeltoft-Hansen, 2011b). Como han señalado diversos autores, han sido precisamente las políticas de externalización, diseñadas para controlar la inmigración irregular (Zaiotti, 2016), las que han tenido un efecto más sobresaliente en las limitaciones de acceso al asilo. ${ }^{9}$

En España este impedimento de acceso al territorio se vuelve aún más nítido en frontera. La colaboración española con Marruecos como parte de la externalización de su política migratoria ha tenido un fuerte impacto en el control preventivo, espacial y físico, de los potenciales solicitantes de asilo que han intentado acceder a Ceuta y Melilla a través de sus perímetros fronterizos o a las provincias costeras del Estrecho y el Mar de Alborán. Estas limitaciones han sido especialmente graves en el caso de los nacionales procedentes de los países del África subsahariana porque para poder acceder a territorio español antes debían evitar la vigilancia terrestre y marítima de la gendarmería marroquí. ${ }^{10}$ Ello les ha abocado a intentar el acceso a través de estrategias de cruce fronterizo que han puesto en peligro su vida y su integridad física, como el salto de las vallas, el acceso a nado, en embarcaciones u ocultos en los vehículos que cada día atraviesan la frontera. Un elemento adicional ha sido la práctica reactiva de devolución inmediata a territorio marroquí conocida habitualmente en la literatura

\footnotetext{
${ }^{7}$ En palabras de este autor mientras que el derecho internacional en materia de refugio ha impuesto la obligación de la aplicación del principio de "no devolución" (non-refoulement), las "políticas de no entrada" se han basado en el compromiso de impedir la llegada de refugiados (Hathaway, 1992).

${ }^{8}$ Como también ha sostenido Agier, la acción de los países europeos ha transitado de la "responsabilidad de proteger" a la "externalización de la responsabilidad de protección" (Agier, 2013), un aspecto que incide precisamente en el "protection lite" al que hacía referencia Gammeltoft-Hansen (2011b).

${ }^{9}$ Como ha indicado Fitzgerald recientemente, los Estados han "evitado" el régimen de asilo a través de una manipulación de la territorialidad conformado por una arquitectura de la repulsión para mantener a los solicitantes alejados de los espacios donde pueden pedir protección (Fitzgerald, 2019, p. 6).

${ }^{10}$ Con esta rotundidad lo expresaba un técnico de una organización social que trabaja en Melilla durante una de las entrevistas: "La frontera es infranqueable para los africanos... ¿Cómo van a aproximarse? La gendarmería marroquí no les deja... por lo que la entrada irregular es la única escapatoria... En los africanos es imposible acceder al asilo en la frontera" (entrevista a un presidente de una oNG que trabaja en la asistencia a inmigrantes en Melilla, mayo de 2016).

Un jurista que trabaja en la frontera sur también lo resume de forma contundente:

el colectivo africano, independientemente del país que sea y pese a los mandatos de ACNUR referidos a situaciones concretas en países africanos, parece que solo se trata de inmigrantes económicos, y consiguientemente el acceso al asilo en frontera es imposible, les está vetado. Les está vetado, ¿por qué?... por la externalización de la frontera sur por parte de España (entrevista a abogado del Colegio de Abogados de Melilla, junio de 2016).
} 
como devoluciones en caliente ${ }^{11}$ (Martínez-Escamilla, 2017; López-Sala, 2020). Estas han sido objeto de amplias controversias políticas y jurídicas ya que, en la práctica, han impedido el acceso al procedimiento de asilo a través de un rechazo directo en frontera que ha dejado al margen las garantías de protección y que ha supuesto la vulneración del principio de non-refoulement. ${ }^{12}$

El etiquetado de la migración que trascurre por la frontera sur como un flujo meramente económico y por lo tanto como no merecedor de recibir protección internacional no solo ha resultado en el desarrollo de prácticas que han impedido su acceso al territorio, sino que ha auspiciado mecanismos restrictivos de acceso al procedimiento que son también mencionados por los teóricos del paradigma disuasorio. En este sentido, el instrumento más explícito fue la introducción en la ley española de refugio de 1994 del denominado "procedimiento de inadmisión a trámite"13 (Gil-Bazo, 1998; Fullerton, 2005), un procedimiento acelerado de escrutinio previo realizado por la administración que posibilitaba o no el acceso al sistema y que supuso en la práctica un serio obstáculo a través de una interpretación muy reducida de las solicitudes consideradas fundadas. Este mecanismo que impedía, de forma retroactiva a los refugiados ya presentes en el territorio "acceder a la puerta del procedimiento" (Vedsted-Hansen, 1999) actuó, en opinión de algunos especialistas y de los técnicos entrevistados durante nuestro trabajo de campo, como un mecanismo disuasorio, que en última instancia terminó por afectar al número de solicitudes de protección que disminuyeron de forma considerable en la segunda mitad de la década de 1990 y se mantuvieron en niveles muy bajos durante la primera década del siglo

${ }^{11}$ Por expulsión o devolución en caliente se entiende:

la actuación de las Fuerzas y Cuerpos de Seguridad del Estado consistente en la entrega a las autoridades marroquíes por vía de hecho de ciudadanos extranjeros que han sido interceptados en zona de soberanía española sin seguir el procedimiento establecido legalmente, ni cumplir las garantías internacionalmente reconocidas (Martínez-Escamilla 2017, p. 60).

Según Martínez-Escamilla esta expresión es aplicable tanto si estas prácticas recaen sobre personas interceptadas saltando las vallas fronterizas, como si estas han accedido a las ciudades de Ceuta y Melilla por mar o alcanzado alguna de las islas de soberanía española situadas frente a las costas de Marruecos.

${ }^{12}$ En el ámbito político ha supuesto importantes enfrentamientos entre distintos gobiernos conservadores del Partido Popular, los grupos de la oposición, la sociedad civil, expertos y los organismos internacionales que han criticado y condenado no solo estas prácticas, sino las más recientes reformas jurídicas para intentar dar amparo legal a este tipo de devoluciones, aspectos que han sido ampliamente señalados por los entrevistados durante nuestro trabajo de campo (véase también Martínez-Escamilla, 2017; López-Sala, 2020). La controversia jurídica ha tenido además un amplio alcance con importantes implicaciones para el conjunto de la política fronteriza europea. En primer lugar, a través de la sentencia del Tribunal Europeo de Derechos Humanos (N. D and N. T v. Spain (nos.8675/15 and 8697/15) de octubre de 2017 (Sánchez-Tomás, 2018; Solanes, 2017) que condenó a España por vulnerar el artículo 4 del Convenio Europeo de Derechos Humanos al indicar que en estas devoluciones los migrantes no son susceptibles de ningún procedimiento de identificación, ni tienen la oportunidad de expresar su deseo de solicitar asilo. Más recientemente y en una dirección contraria la controvertida sentencia de febrero de 2020 del mismo tribunal que se desdice de los anteriores presupuestos sosteniendo que España no vulneró dicho artículo del Convenio Europeo (para mayor detalle véase Carrera, 2020).

${ }^{13}$ Este procedimiento de inadmisión a trámite es una peculiaridad española y portuguesa entre los países europeos que conforman la frontera sur europea (Fullerton, 2005), si bien todos ellos han llegado a desarrollar a lo largo de las últimas dos décadas mecanismos específicos a la hora de la gestión de las solicitudes en frontera, como procedimientos rápidos o el más reciente hot spots approach (Casolari, 2015). La secuencia temporal de la aplicación de otras medidas, como la externalización o las devoluciones en caliente, se adelantó en el caso español respecto a sus vecinos europeos (Godenau \& López-Sala, 2016). 
XxI. ${ }^{14}$ Además, la reforma legal introducía un procedimiento acelerado en frontera que exigía que los solicitantes permanecieran en dependencias habilitadas en las propias instalaciones fronterizas hasta que las autoridades determinaran su admisión, o no, al procedimiento. ${ }^{15}$ Las dificultades de acceso al procedimiento en frontera se agravaron aún más con la última reforma legal de 2009 que estableció un sistema que permitía al Ministerio del Interior no solo inadmitir a trámite la solicitud, sino denegar la solicitud de asilo de forma acelerada en un plazo máximo de cuatro días (Sánchez-Legido, 2009; García-Mahamut \& Galparsoro, 2010; Valles, 2016).

Estas limitaciones legales se han compaginado con elementos procedimentales que han producido lo que podemos denominar un "vacío infraestructural" para el acceso a la condición de refugiado en la frontera sur española. La consideración de que las causas económicas eran las únicas razones que motivaban la movilidad de los migrantes que trascurren por estos corredores ha devenido en la ausencia, en la práctica, de infraestructura, procedimientos y recursos humanos y materiales que permitieran realizar la solicitud de asilo, tanto en la llegada a costa como en los puestos habilitados de cruce (véase Solanes, 2014), un elemento reiteradamente expuesto por nuestros entrevistados como una de las principales causas del bajo número de solicitudes en frontera. No debemos olvidar que no existe un protocolo de identificación de personas que puedan necesitar protección internacional en el cruce irregular por los perímetros terrestres de la frontera periférica, incluidos menores no acompañados y víctimas de trata; y que hasta septiembre de 2014 en las ciudades de Ceuta y Melilla no existían oficinas específicas para tramitar estas solicitudes en los puestos habilitados de entrada de Tarajal y Beni Enzar. ${ }^{16}$ Desde hace más de una década esta situación había sido denunciada por numerosas organizaciones internacionales y no gubernamentales (Amnesty International, 2005; CEAR, 2012) y había sido objeto de informes y recomendaciones realizados, entre otros, por el Defensor del Pueblo y la Comisión Europea contra el Racismo y la Intolerancia (ECRI) del Consejo de Europa (Defensor del Pueblo, 2005; ECRI, 2005). En la práctica, esta situación supuso que hasta 2014 las peticiones de asilo solo pudieran ser efectuadas una vez dentro del territorio de ambas ciudades, habitualmente en los denominados Centros de Estancia Temporal

\footnotetext{
${ }^{14}$ De acuerdo con los datos proporcionados por el Ministerio del Interior, entre 1994 y $2009,70 \%$ de las solicitudes fueron inadmitidas a trámite (véase también Izquierdo, 2004).

${ }^{15} \mathrm{Al}$ respecto Valles señala:

Esta disposición fue recurrida por el Defensor del Pueblo ante el Tribunal Constitucional que sin embargo desestimó el recurso al considerar que no se trataba de una detención, sino de una retención, por lo que no violaba la carta magna (Valles, 2016, p. 231).

${ }^{16}$ Es ilustrativo que, aunque las leyes españolas de asilo lo contemplaban desde mediados de la década de 1990, hasta 2014 no se realizó ninguna solicitud de asilo en las fronteras terrestres de Ceuta y Melilla. De hecho, desde la apertura de una oficina en el puesto fronterizo de Tarajal (Ceuta) en marzo de 2015 no se ha realizado ninguna solicitud de protección internacional. Según los datos del ACNUR, entre octubre de 2014 y el 31 de julio de 2017, 9760 personas solicitaron protección internacional en el puesto fronterizo de Beni Enzar, en Melilla. De los solicitantes, $90 \%$ eran ciudadanos sirios y el resto personas originarias de Marruecos, Yemen y Palestina. En 2018 se mantuvo esta tendencia según los informes más recientes de la Comisión Española de Ayuda al Refugiado (CEAR). A lo largo de 2018, se realizaron 2800 solicitudes de refugio en el puesto fronterizo de Beni Enzar, principalmente de personas procedentes de Siria, Palestina y Yemen (CEAR, 2018, 2019).
} 
(Cetis) ${ }^{17}$ Es importante destacar, además, que la apertura de estas dos oficinas en los puestos habilitados para el cruce en las ciudades de Ceuta y Melilla respondió, en gran parte, a la presión internacional y del ACNUR ante el incremento de la llegada de familias sirias a Melilla desde mediados de 2013, en pleno debate europeo sobre el sistema de asilo.

Sin embargo, las dificultades de los solicitantes de asilo no terminan una vez en el interior del territorio de ambas ciudades, lo que da cuenta de las especificidades españolas en la aplicación del denominado paradigma disuasorio. Tras la última reforma de la ley de asilo en 2009, la Secretaría de Estado de Seguridad empezó a aplicar un procedimiento administrativo que restringe y somete a serias limitaciones la movilidad de los solicitantes de asilo que desean desplazarse al territorio español peninsular. Esta limitación de la movilidad intraterritorial de los solicitantes de asilo ha sido justificada con base en el estatuto especial de ambas ciudades autónomas en el acuerdo de Schengen; acuerdo que permite que España se reserve el derecho a mantener los controles documentales en las conexiones entre estas dos ciudades y la península (Solanes, 2014; González-García, 2015; Valles, 2016; López-Sala, 2020). De acuerdo con la información de la Secretaría de Estado de Seguridad, la documentación de admisión a trámite del procedimiento de asilo —una tarjeta identificadora entregada a los solicitantes- no puede considerarse válida como acreditación para viajar, lo que ha supuesto la denegación de los permisos de viaje de los solicitantes que quieren desplazarse a otras zonas del territorio nacional. Esta barrera administrativa, que en realidad produce una "cautividad burocrática" de los solicitantes de asilo ha transformado a Ceuta y Melilla en lo que algunos migrantes califican como "una cárcel dorada" (López-Sala, 2015b) porque la solicitud del estatuto de asilo implica su inmovilización involuntaria en estas ciudades, una retención física que puede extenderse meses e incluso años, hasta que esta solicitud es resuelta. Esta seria limitación de la libertad de circulación de los solicitantes de asilo puede considerarse una estrategia disuasoria adicional dentro del complejo puzle del control migratorio (López-Sala, 2015a), llevando a muchas personas necesitadas de protección internacional a no solicitar este estatuto para así poder entrar en lo que se denomina el programa de $\operatorname{traslados}^{18} \mathrm{y}$ acceder a territorio europeo continental. ${ }^{19}$

\footnotetext{
${ }^{17}$ Los Cetis son los centros de estancia temporal en régimen semiabierto y bajo la dirección del Ministerio de Asuntos Sociales y Trabajo en el que los inmigrantes y solicitantes de asilo en Ceuta y Melilla residen temporalmente hasta que su situación legal es resuelta.

${ }^{18}$ El programa de traslados humanitarios, que carece de regulación legal, es un mecanismo que empezó a emplearse para evitar la falta de recursos y la saturación de los centros de acogida ante el incremento de llegadas a mediados de la década de los 2000 tanto en Ceuta y Melilla como en Canarias. A partir de 2015, en el caso de los solicitantes de asilo y refugio empiezan a aplicarse los traslados humanitarios, y casi en exclusiva, a los nacionales de Siria. De ahí que las organizaciones sociales hayan denunciado la discriminación por nacionalidad en la aplicación de estas medidas (CEAR, 2018; Amnistía Internacional, 2016).

${ }^{19}$ Así lo expresaba uno de nuestros entrevistados en Melilla:

porque no había peticiones... porque no había problema de asilo en España, pero claro que hay... o sea, en los demás países había miles de solicitantes de asilo y España nunca tenía nada, entonces ¿Cómo es posible, cómo puede ser? ... En España no se pide asilo (...) no había solicitantes de asilo pues porque era un castigo. Pedir asilo suponía recibir un castigo por parte de la Administración, entonces la gente no pedía asilo. No es porque no hubiera... (entrevista a un presidente de una ONG que trabaja en la asistencia a inmigrantes en Melilla, mayo de 2016).
} 
Estas medidas de inmovilización selectiva contravienen la ley española de asilo y vulneran el principio de igualdad de trato como han denunciado en los últimos años el ACNur y el Defensor del Pueblo (United Nations General Assembly, 2013; Defensor del Pueblo, 2013, 2016). El Defensor del Pueblo señalaba, además, en su informe de 2016 que "estas prácticas afectaban a la credibilidad del sistema de protección español y suponían el agravamiento de la vulnerabilidad de estas personas" (Defensor del Pueblo, 2016, p. 52). A pesar de que varias resoluciones judiciales han declarado esta práctica contraria a la legalidad, ${ }^{20}$ continúa aplicándose en la actualidad.

\section{Conclusiones. "Prácticas de imposibilidad" y "dinámicas de ausencia"}

El etiquetado de la inmigración que trascurre por la frontera sur española, fundamentalmente africana, como un flujo motivado exclusivamente por razones económicas y laborales ha supuesto su categorización como una movilidad voluntaria. Esto ha tenido enormes implicaciones en la modulación de los regímenes de inmigración y de refugio en los contornos territoriales españoles y, en particular, en su frontera marítima y terrestre periférica. Este etiquetado, conformado y consolidado a lo largo de las tres últimas décadas, que ha convertido a los migrantes africanos en potenciales falsos solicitantes de asilo (o solicitantes fraudulentos) ha producido una regulación y una intervención política y administrativa basada en la prevención, la contención y la retención que ha invisibilizado, primero, e impedido, después, el deber de dar respuesta a sus necesidades de protección. En la primera parte del artículo se han presentado los efectos en el régimen de movilidad de esta categorización y estereotipación de la que habla la teoría del etiquetado y sus efectos sobre el régimen del refugio en esta franja de la frontera sur europea. El análisis del caso español ha permitido caracterizar los mecanismos de limitación del acceso a protección de los potenciales solicitantes de asilo y muestra rasgos que están en sintonía con lo apuntado en otros contextos geográficos por los teóricos del paradigma disuasorio. Entre otros se han destacado las dificultades de acceso al territorio, a través de las bien conocidas políticas de externalización con Marruecos o de las tristemente famosas devoluciones en caliente. Así mismo se han destacado las enormes dificultades de acceso al procedimiento, a través de la aplicación durante más de una década de la denominada admisión a trámite. También ha servido para destacar algunos elementos distintivos observados en el caso español, entre otros, la limitación de movilidad intraterritorial de los solicitantes que nos ha permitido hablar de una forma de "cautiverio burocrático" de estos solicitantes, aún después de su acceso al territorio y al procedimiento. Este conjunto de acciones ha devenido en una extrema dificultad de acceso a protección mostrando unas prácticas de imposibilidad que tienen su expresión más fehaciente en la escasez de solicitudes de asilo en frontera. Los marcos regulatorios y políticos se han compaginado con la ausencia de elementos procedimentales que han producido lo que hemos denominado un "vacío infraestructural". La consideración de que las

\footnotetext{
${ }^{20}$ Entre ellas las sentencias del Tribunal Superior de Justicia de Andalucía del 13 de enero de 2011 y de febrero de 2012 y del Tribunal Superior de Justicia de Madrid del 11 de mayo de 2015 y del 30 de octubre de 2018.
} 
causas económicas eran las únicas razones que motivaban la movilidad de los migrantes que trascurren por estos corredores ha devenido en la ausencia, en la práctica, de infraestructura, procedimientos y recursos humanos y materiales que permitieran realizar la solicitud de asilo, tanto en la llegada a costa como en los puestos habilitados de cruce.

\section{Agradecimientos}

Este artículo ha sido desarrollado en el contexto del proyecto MiND ("Actores no estatales del control migratorio en España”), financiado por el Ministerio Español de Ciencia e Innovación (CSO2014-53680-P).

\section{Referencias}

Agier, M. (2013). Managing the Undesirables. Refugee Camps and Humanitarian Government. Policy.

Amnesty International. (2005, 19 de junio). Spain: The Southern Border. The State Turns its Back on the Human Rights of Refugees and Migrants (AI Index: EUR 41/008/2005).

Amnistía Internacional. (2016, 29 de noviembre). Ceuta y Melilla. Un territorio sin derechos para personas migrantes y refugiadas.

Alto Comisionado de las Naciones Unidas para los Refugiados (ACNUR). (2009, abril). Protección de los refugiados y migración internacional: revisión del papel de ACNUR en las Islas Canarias (España). Autor.

Apostolova, R. (2015, 14 de septiembre). Of Refugees and Migrants: Stigma, Politics, and Boundary Work at the Borders of Europe. American Sociological Association, culture section web site and newsletter. https:/ / asaculturesection.org/2015/09/14/ of-refugees-and-migrants-stigma-politics-and-boundary-work-at-the-borders-ofeurope/

Becker, M. (2014). The Discourse About Legal Protection for Environmental Refugees: Reconstructing Categories - Rethinking Processes. En F. Gesing, J. Herbeck \& S. Klepp (Eds.), Denaturalising Climate Change: Migration, Mobilities and Space (pp. 77-85). Universidad de Bremen.

Bivand Erdal, M. \& Oeppen, C. (2018). Forced to leave? the discursive and analytical significance of describing migration as forced and voluntary. Journal of Ethnic and Migration Studies, 44(6), 981-998.

Bodelón, G. (1993). La Oficina de Asilo y Refugio (OAR). Revista Minusval, 87, 47-48.

Carrera, S. (2020, marzo). The Strasbourg Court Judgement N.D. and N.T. v Spain. A Carte Blanche to Push Backs at EU External Borders? EUI Working Papers.

Casolari, F. (2015). The EU's hotspot approach to managing the migration crisis: a blind spot for international responsibility? The Italian Yearbook of International Law, 25, 109-134.

Comisión Española de Ayuda al Refugiado (CEAR). (2007). La situación de los refugiados en España. Entimema. 
Comisión Española de Ayuda al Refugiado (CEAR). (2012). Informe 2012. La situación de las personas refugiadas en España. Madrid.

Comisión Española de Ayuda al Refugiado (CEAR). (2018). Informe 2018. Las personas refugiadas en España y en Europa. Madrid. https://www.cear.es/wp-content/ uploads/2018/06/Informe-CEAR-2018.pdf

Comisión Española de Ayuda al Refugiado (CEAR). (2019). Informe 2019. Las personas refugiadas en España y en Europa. Madrid. https://www.cear.es/wp-content/ uploads/2019/07/INFORME_CEAR_2019.pdf

Collyer, M. (2019). From preventive to repressive: the changing use of development and humanitarism to control migration. En K. Mitchell, R. Jones \& J. Flury (Eds.), Critical Geographies of Migration (pp. 170-181). Edward Elgar.

Crawley, H. \& Skleparis, D. (2018). Refugees, migrants, neither, both: categorical fetishism and the politics of bounding in Europe's 'migration crisis'. Journal of Ethnic and Migration Studies, 44(1), 48-64.

Defensor del Pueblo. (2005). Informe sobre Asistencia Jurídica de los Extranjeros en España. Autor.

Defensor del Pueblo. (2013). Informe anual 2013 y debates en las Cortes Generales. Autor. https://www.defensordelpueblo.es/wp-content/uploads/2015/05/2013_Informe_Anual_Vol_I_Informe_2013.pdfDefensor del Pueblo. (2016). Estudio sobre el asilo en España. La protección internacional y los recursos de acogida del sistema. Autor.

Escobar-Hernández, C. (1992, enero). Asylum and refugee status in Spain. International Journal of Refugee Law, 4(1), 57-70.

Espada Ramos, M. L. \& Moya Escudero, M. (1985). La ley reguladora del asilo y la condición de refugiado del 26 de marzo de 1984: ¿nacionalismo o internacionalismo? Revista de Estudios Internacionales 6(1), 73-110.

European Commission against Racism and Intolerance (ECRI). (2005, 14 de junio). Tercer Informe sobre España. https://rm.coe.int/third-report-on-spain-spanish-translation-/16808b56c5

Ferrer-Gallardo, X. (2008). The Spanish-Moroccan border complex. Processes of geopolitical, functional and symbolic rebordering. Political Geography, 27(3), 301-321.

Ferrer-Gallardo, X. \& Gabrielli, L. (2018). Estados de excepción en la excepción del Estado. Ceuta y Melilla. Icaria.

Fitzgerald, D. (2019). Refuge Beyond Reach: How Rich Democracies Repel Asylum Seekers. Oxford University Press.

Fullerton, M. (2005). Inadmissible in Iberia: The fate of asylum seekers in Spain and Portugal. International Journal of Refugee Law, 17(4), 659-687.

Gammeltoft-Hansen, T. (2011a). Access to Asylum. International Refugee Law and the Globalisation of Migration Control. Cambridge University Press.

Gammeltoft-Hansen, T. (2011b). Outsourcing asylum: The advent of "protection lite". En L. Bialasewitcz (Ed.), Europe in the World: EU Geopolitics and the Making of European Space (pp. 129-140). Ashgate.

Gammeltoft-Hansen, T. \& Tan, N. (2017). The end of the deterrence paradigm? Future directions for global refugee policy. Journal of Migration and Human Security, $5(1), 28-56$. 
Gammeltoft-Hansen, T. \& Vedsted-Hansen, J. (Eds.). (2016). Human Rights and the Dark Side of Globalisation. Transnational Law Enforcement and Migration Control. Routledge.

Garcés-Mascareñas, B. (2019). Ser o no ser. Deficiencias en el sistema estatal de acogida. Notes Internacionals СIDOB, 214, 1-5.

Garcés-Mascarenas, B. \& Moreno-Amador, G. M. (2019). The multilevel governance of refugee reception policies in Spain. CEASEVAL Research on the Common European Asylum System, (18). http://ceaseval.eu/publications/WP3_Spain.pdf.

García-Mahamut, R. \& Galparsoro, J. (2010). Régimen Jurídico del Derecho de Asilo en la Ley 12/2009. Centro de Estudios Políticos y Constitucionales.

Gil-Bazo, M. T. (1998). The role of Spain as a gateway to the Schengen Area. Changes in the asylum law and their implications for human rights. International Journal of Refugee Law 10(1,2), 214-229.

Giuffré, M \& Moreno-Lax, V. (2017). The Raise of Consensual Containment: From Contactless Control to Contactless Responsability for Forced Migration Flows. En S. Juss (Ed.), Research Handbook on International Refugee Law (pp. 82-108). Edward Elgar.

Godenau, D. (2014). Irregular maritime immigration in the Canary Islands: Externalization and communautarisation in the social construction of borders. Journal of Immigrant and Refugee Studies, 12(2), 123-142.

Godenau, D. \& López-Sala, A. (2016). Multi-Layered Migration Deterrence and Technology in Spanish Maritime Border Management, Journal of Borderland Studies, 31(2), 151-169.

González-García, J. V. (2015). Expulsiones "en caliente", devoluciones y petición de asilo en Ceuta y Melilla, Revista de Administración Pública, 196, 309-329.

Hathaway, J. (1992). The Emerging Politics of Non-Entrée. Refugees, 91, 40- 41.

Hathaway, J. \& Gammeltoft-Hansen, T. (2015). Non-refoulement in a world of cooperative deterrence. Columbia Journal of Transnational Law 53(2), 235-285.

Hirsch, A. L. (2017). The Borders Beyond the Border: Australia's Extraterritorial Migration Controls. Refugee Survey Quarterly 36(3), 48-80.

Iglesias, J. \& Estrada, C. (2018). Birds of passage? La integración social de la población refugiada en España. Iberoamerican Journal of Development Studies, 7(1), 144-167. https://doi.org/10.26754/ojs_ried/ijds.279

Iglesias, J., Urrutia, G., Buades, J., Estrada, C. \& Vicente, T. (2018). ¿Acoger sin integrar? El sistema de acogida y las condiciones de integración de personas solicitantes y beneficiarias de protección internacional en España. Universidad Pontificia de Comillas/Servicio Jesuita a los Refugiados/Universidad de Deusto.

Izquierdo, A. (2001). Panorama de la inmigración en España al alba del siglo xxi. Mediterráneo Económico, 1, 247-264.

Izquierdo, A. (2004). Cambios en la inmigración a resueltas de la política restrictiva del gobierno español (The Center for Comparative Immigration Studies University of California). Working Paper 109.

Izquierdo, A., López-Lera, D. \& Martínez-Buján, R. (2003). The favorites of the twenty-first century: Latin American immigration in Spain. Studi Emigrazione, (149), 98-124. 
Janmyr, M. \& Mourad, L. (2018). Modes of ordering: labelling, classification and categorization in Lebanon's refugee response. Journal of Refugee Studies, 31(4), 544565. https://doi.org/10.1093/jrs/fex042

Jubany, O. (2002). The state of welfare for asylum seekers and refugees in Spain. Critical Social Policy, 22(3), 415-435.

Jubany, O. (2011). Constructing truths in a culture of disbelief. Understanding asylum screening from within. International Sociology, 26(1), 74-94.

Leach, M. \& Zamora, A. (2006). Illegals/Ilegales: Comparing antiimmigrant/anti-refugees discourses in Australia and Spain. Journal of Iberian and Latin American Research, 12(1), 51-64.

López-Sala, A. (2015a). Exploring dissuasion as a (geo)political instrument in irregular migration control at the southern Spanish maritime border. Geopolitics, 20(3), 513-534.

López-Sala, A. (2015b). La inmigración irregular en Ceuta y Melilla en 2014. Prácticas de control y debate público. En J. Arango, D. Moya, J. Oliver \& E. Sánchez-Montijano (Eds.), Flujos cambiantes, atonía institucional. Anuario de la inmigración en España (pp. 170-191). сіDов.

López-Sala, A. (2020). Keeping up appearances. Dubious legality and migration control at the peripheral borders of Europe. The cases of Ceuta and Melilla. En S. Carrera \& M. Stefan (Eds.), Fundamental Rights Challenges in Border Controls and Expulsion of Irregular Immigrants in the European Union (pp. 25-42). Routledge.

López-Sala, A. \& Godenau, D. (2016). Integrated border management and irregular migration at the South European-North African border: The case of Spain. En R. Bossong \& H. Carrapico (Eds.). EU Borders and Shifting Internal Security: Technology, Externalization and Accountability (pp. 81-100). Springer.

López-Sala, A. \& Godenau, D. (Coords.). (2017a). Estados de contención, Estados de detención. El control de la inmigración irregular en España. Anthropos.

López-Sala, A. \& Godenau, D. (2017b). Controlling irregular immigration at the European union's southern maritime border. An emerging system driven by "migration emergencies". Journal of Mediterranean Knowledge, 2(1), 17-35

Martínez-Escamilla, M. (2017). Fronteras sin derechos. Las "devoluciones en caliente". En A. López-Sala \& D. Godenau (Eds.), Estados de contención, Estados de detención. El control de la inmigración irregular en España (pp. 54-74). Anthropos.

McFadyen, G. (2016). The language of labelling and the politics of hospitality in the British asylum system. The British Journal of Politics and International Relations, 18(3), 599-617.

McMahon, S. \& Sigona, N. (2018). Navigating the Central Mediterranean in a Time of 'Crisis': Disentangling Migration Governance and Migrant Journeys. Sociology, 52(3), 497-514.

Moncrieffe, J. (2007). Labelling, power and accountability: how and why "our" categories matter. En J. Moncrieffe \& R. Eyben (Eds.), The Power of Labelling. How People are Categorized and Why it Matters (pp. 1-17). Earthscan.

Pérez-Sola, N. (2011). El derecho de asilo y la condición de refugiado. Balance de 26 años de desarrollos legislativos. Derechos y Libertades, 15(25), 269-308. 
Sajjad, T. (2018). What's in a name? 'Refugees', 'migrants' and the politics of labelling. Race and Class, 60(2), 40-62.

Sánchez-Legido, A. (2009). Entre la obsesión por la seguridad y la lucha contra la inmigración irregular. A propósito de la nueva Ley de Asilo. Revista Electrónica de Estudios Internacionales, 18, 1-32.

Sánchez-Tomas, J. (2018). Las "devoluciones en caliente" en el Tribunal Europeo de Derechos Humanos, Revista Española de Derecho Europeo, 65, 101-135.

Santaolaya, P. (2006). El sistema común de asilo y su recepción en España. Revista de Derecho Migratorio y Extranjería, 12, 83-89.

Sigona, N. (2017). The contested politics of naming in Europe's "refugee crisis". Ethnic and Racial Studies, 41(3), 456-460.

Solanes, A. (2014). Derechos Humanos y Asilo. Sobre las deficiencias del SECA y la regulación jurídica española. Anuario Facultad de Derecho-Universidad de Alcalá, 7, 181-210.

Solanes, A. (2017). Contra la normalización de la ilegalidad. La protección judicial de los extranjeros frente a las expulsiones colectivas y las devoluciones "en caliente”. Cuadernos Electrónicos de Filosofía del Derecho, 36, 195-225. https://doi. org/10.7203/CEFD.36.11269

Stepputat, F. \& Nyberg-Sorensen, N. (2014). Sociology and Forced Migration. En E. Fiddian-Qasmiyeh, G. Loescher, K. Long \& N. Sigona (Eds.), The Oxford Handbook of Refugee and Forced Migration Studies (pp. 86-98). Oxford University Press.

United Nations General Assembly. (2013). Report of the special rapporteur on contemporary forms of racism, racial discrimination, xenophobia and related intolerance, Mutuma Ruteere, A/HRC/23/56/Add.2

Valles, M. (2016). ¿Vallas al asilo? Apuntes sobre el sistema de protección en España. Anиатіо СIDOB de la Inmigración, 226-245

Vedsted-Hansen, J. (1999). Europe's response to the arrival of asylum seekers: refugee protection and immigration control. UNHCR.

Vives, L. (2011). White Europe: an alternative reading of the Southern EU border. Geopoliticas, 2(1), 51-70.

Vradis, A., Papada, E., Painter, J. \& Papoutsi, A. (2019). New Borders. Hotspots and the European Migration Regime. Pluto Press.

Wood, G. (1985). The politics of development policy labelling. Development and Change, 16(3), 347-373.

Zaiotti, R. (Ed.). (2016). Externalizing Migration Management. Europe, North America and the Spread of "Remote Control" Practices. Routledge.

Zetter, R. (1991). Labelling refugees: Forming and transforming a bureaucratic identity. Journal of Refugee Studies, 4(1), 39-62. https://doi.org/10.1093/jrs/4.1.39

Zetter, R. (2007). More labels, fewer refugees: remaking the refugee label in an era of globalization. Journal of Refugee Studies, 20(2), 172-192. 
Ana López-Sala

Española. Doctora en sociología por la Universidad Complutense de Madrid y Científica Titular en el Instituto de Economía, Geografía y Demografía del csic, España. Líneas de investigación: estudio comparado de las políticas migratorias y las dinámicas sociales y políticas relacionadas con la inmigración irregular. En la actualidad es board del Research Committee (Sociology of Migration) de la International Sociological Association (ISA). Publicación reciente: López-Sala, A. \& Godenau, D. (coord.). (2017). Estados de contención, Estados de detención. El control de la inmigración irregular en España. Bercelona: Anthropos.

Gracia Moreno-Amador

Española. Licenciada en sociología y en trabajo social (UCM, UCLM) y máster en estudios migratorios y estudios de cooperación (UPCO). Es investigadora predoctoral en el Instituto de Economía, Geografía y Demografía del csic donde ha realizado una tesis doctoral sobre políticas de gestión del refugio en España (2020). En la actualidad es profesora en el máster de estudios de la Universidad Pontificia. Líneas de investigación: políticas de refugio y de ciudadanía de refugiados en España y en Europa. Publicación reciente: Garcés Mascareñas, B. \& Moreno-Amador, G. (2019). The Multilevel Governance of Refugee Reception Policies in Spain. En Ceaseval Research on the Common European Asylum System, (18). 